NUC-MINN-2001/11-T

September 2001

\title{
HIGH TEMPERATURE MATTER AND GAMMA RAY SPECTRA FROM MICROSCOPIC BLACK HOLES
}

\author{
R. G. Daghigh and J. I. Kapusta \\ School of Physics and Astronomy, University of Minnesota \\ Minneapolis, MN 55455, USA
}

\begin{abstract}
The relativistic viscous fluid equations describing the outflow of high temperature matter created via Hawking radiation from microscopic black holes are solved numerically for a realistic equation of state. We focus on black holes with initial temperatures greater than $100 \mathrm{GeV}$ and lifetimes less than 6 days. The spectra of direct photons and photons from $\pi^{0}$ decay are calculated for energies greater than $1 \mathrm{GeV}$. We calculate the diffuse gamma ray spectrum from black holes distributed in our galactic halo. However, the most promising route for their observation is to search for point sources emitting gamma rays of ever-increasing energy.
\end{abstract}

\section{Introduction}

Hawking radiation from black holes [1] is of fundamental interest because it relies on the application of relativistic quantum field theory in the presence of the strong field limit of gravity, a situation that could potentially be observed. It is also of great interest because of the temperatures involved. A black hole with mass $M$ radiates thermally with a Hawking temperature $T_{H}=m_{\mathrm{P}}^{2} / 8 \pi M$ where $m_{\mathrm{P}}=G^{-1 / 2}=1.22 \times 10^{19} \mathrm{GeV}$ is the Planck mass. (Units are $\hbar=c=k_{\mathrm{B}}=1$.) In order for the black hole to evaporate rather than accrete it must have a temperature greater than that of the present-day black-body radiation of the universe of $2.7 \mathrm{~K}=2.3 \times 10^{-4} \mathrm{eV}$. This implies that $M$ must be less than $1 \%$ of the mass of the Earth. Such small black holes most likely would have been formed primordially; there is no other mechanism known to form them. As the black hole radiates, its mass decreases 
and its temperature increases until $T_{H}$ becomes comparable to the Planck mass, at which point the semi-classical calculation breaks down and the regime of full quantum gravity is entered. Only in two other situations are such enormous temperatures achievable: in the early universe and in central collisions of heavy nuclei like gold or lead. Even then only about $T=500 \mathrm{MeV}$ is reached at the RHIC (Relativistic Heavy Ion Collider) just completed at Brookhaven National Laboratory and $T=1 \mathrm{GeV}$ is expected at the LHC (Large Hadron Collider) at CERN to be completed in 2006. Supernovae and newly formed neutron stars only reach temperatures of a few tens of $\mathrm{MeV}$. To set the scale from fundamental physics, we note that the spontaneously broken chiral symmetry of QCD gets restored in a phase transition/rapid crossover at a temperature around $170 \mathrm{MeV}$, while the spontaneously broken gauge symmetry in the electroweak sector of the standard model gets restored in a phase transition/rapid crossover at a temperature around $100 \mathrm{GeV}$. The fact that temperatures of the latter order of magnitude will never be achieved in a terrestrial experiment should motivate us to study the fate of microscopic black holes during the final days, hours and minutes of their lives when their temperatures have risen to $100 \mathrm{GeV}$ and above. In this paper we shall focus on Hawking temperatures greater than $100 \mathrm{GeV}$. The fact that microscopic black holes have not yet been observed [2] should not be viewed as a deterrent, but rather as a challenge for the new millennium!

There is some uncertainty over whether the particles scatter from each other after being emitted, perhaps even enough to allow a fluid description of the wind coming from the black hole. Let us examine what might happen as the black hole mass decreases and the associated Hawking temperature increases.

When $T_{H} \ll m_{\mathrm{e}}$ (electron mass) only photons, gravitons, and neutrinos will be created with any significant probability. These particles will not interact with each other but will be emitted into the surrounding space with the speed of light. Even when $T_{H} \approx m_{\mathrm{e}}$ the Thomson cross section is too small to allow the photons to scatter very frequently in the rarified electron-positron plasma around the black hole. This may change when $T_{H} \approx 100 \mathrm{MeV}$ when muons and charged pions are created in abundance. At somewhat higher temperatures hadrons are copiously produced and local thermal equilibrium may be achieved, although exactly how is an unsettled issue. Are hadrons emitted directly by the black hole? If so, they will be quite abundant at temperatures of order $150 \mathrm{MeV}$ because their mass spectrum rises exponentially (Hagedorn growth as seen in the Particle Data Tables [3]). Because they are so massive they move nonrelativistically and may form a very dense equilibrated gas around the black hole. But hadrons are composites of quarks and gluons, so perhaps quarks and gluon jets are emitted instead? These jets must decay into the observable hadrons on a typical proper length scale of $1 \mathrm{fm}$ and a typical proper time scale of $1 \mathrm{fm} / \mathrm{c}$. This was first studied by MacGibbon and Webber [4] and MacGibbon and Carr [5]. Subsequently Heckler [6] argued that since the emitted quarks and gluons are so densely packed outside the event horizon they are not actually fragmenting into hadrons in vacuum but in something more like a quark-gluon plasma, so perhaps they thermalize. He also argued that QED bremsstrahlung and pair production were sufficient 
to lead to a thermalized QED plasma when $T_{H}$ exceeded $45 \mathrm{GeV}$ [7]. These results are somewhat controversial and need to be confirmed. The issue really is how to describe the emission of wavepackets via the Hawking mechanism when the emitted particles are (potentially) close enough to be mutually interacting. A more quantitative treatment of the particle interactions on a semiclassical level was carried out by Cline, Mostoslavsky and Servant [8]. They solved the relativistic Boltzmann equation with QCD and QED interactions in the relaxation-time approximation. It was found that significant particle scattering would lead to a photosphere though not perfect fluid flow.

Rather than pursuing the Boltzmann transport equation one of us applied relativistic viscous fluid equations to the problem assuming sufficient particle interaction [9]. It was found that a self-consistent description emerges of a fluid just marginally kept in local thermal equilibrium, and that viscosity is a crucial element of the dynamics. The purpose of this paper is a more extensive analysis of these equations and their observational consequences. The plan is as follows. In section 2 we give a brief review of Hawking radiation sufficient for our uses. In section 3 we give the set of relativistic viscous fluid equations necessary for this problem along with the assumptions that go into them. In section 4 we suggest a relatively simple parametrization of the equation of state for temperatures ranging from several $\mathrm{MeV}$ to well over $100 \mathrm{GeV}$. We also suggest a corresponding parametrization of the bulk and shear viscosites. In section 5 we solve the equations numerically, study the scaling behavior of the solutions, and check their physical self-consistency. In section 6 we estimate where the transition from viscous fluid flow to free-streaming takes place. In section 7 we calculate the instantaneous and time-integrated spectra of high energy photons from the two dominant sources: direct and neutral pion decay. In section 8 we study the diffuse gamma ray spectrum from microscopic black holes distributed in our galactic halo. We also study the systematics of gamma rays from an individual black hole, should we be so fortunate to observe one. We conclude the paper in section 9 .

\section{Hawking Radiation}

There are at least two intuitive ways to think about Hawking radiation from black holes. One way is vacuum polarization. Particle-antiparticle pairs are continually popping in and out of the vacuum, usually with no observable effect. In the presence of matter, however, their effects can be observed. This is the origin of the Lamb effect first measured in atomic hydrogen in 1947. When pairs pop out of the vacuum near the event horizon of a black hole one of them may be captured by the black hole and the other by necessity of conservation laws will escape to infinity with positive energy. The black hole therefore has lost energy - it radiates. Due to the general principles of thermodynamics applied to black holes it is quite natural that it should radiate thermally. An intuitive argument that is more quantitative is based on the uncertainty principle. Suppose that we wish to confine 
a massless particle to the vicinity of a black hole. Given that the average momentum of a massless particle at temperature $T$ is approximately $\pi T$, the uncertainty principle requires that confinement to a region the size of the Schwarzschild diameter places a restriction on the minimum value of the temperature.

$$
\pi T \cdot 2 r_{S}>1 / 2
$$

The minimum is actually attained for the Hawking temperature. The various physical quantities are related as $r_{S}=2 M / m_{\mathrm{P}}^{2}=1 / 4 \pi T_{H}$.

The number of particles of spin $s$ emitted with energy $E$ per unit time is given by the formula

$$
\frac{d N_{s}}{d E d t}=\frac{\Gamma_{s}}{2 \pi} \frac{1}{\exp \left(E / T_{H}\right)-(-1)^{2 s}} .
$$

All the computational effort really goes into calculating the absorption coefficient $\Gamma_{s}$ from a relativistic wave equation in the presence of a black hole. Integrating over all particle species yields the luminosity.

$$
L=-\frac{d M}{d t}=\alpha(M) \frac{m_{\mathrm{P}}^{4}}{M^{2}}=64 \pi^{2} \alpha\left(T_{H}\right) T_{H}^{2} .
$$

Here $\alpha(M)$ is a function reflecting the species of particles available for creation in the gravitational field of the black hole. It is generally sufficient to consider only those particles with mass less than $T_{H}$; more massive particles are exponentially suppressed by the Boltzmann factor. Then

$$
\alpha=2.011 \times 10^{-8}\left[4200 N_{0}+2035 N_{1 / 2}+835 N_{1}+95 N_{2}\right]
$$

where $N_{s}$ is the net number of polarization degrees of freedom for all particles with spin $s$ and with mass less than $T_{H}$. The coefficients for spin $1 / 2,1$ and 2 were computed by Page [10] and for spin 0 by Sanchez [11]. In the standard model $N_{0}=4$ (Higgs), $N_{1 / 2}=90$ (three generations of quarks and leptons), $N_{1}=24(\mathrm{SU}(3) \times \mathrm{SU}(2) \times \mathrm{U}(1)$ gauge theory), and $N_{2}=2$ (gravitons). This assumes $T_{H}$ is greater than the temperature for the electroweak gauge symmetry restoration. Numerically $\alpha\left(T_{H}>100 \mathrm{GeV}\right)=4.43 \times 10^{-3}$. Starting with a black hole of temperature $T_{H}$, the time it takes to evaporate/explode is

$$
\Delta t=\frac{m_{\mathrm{P}}^{2}}{3 \alpha\left(T_{H}\right)\left(8 \pi T_{H}\right)^{3}} .
$$

This is also the characteristic time scale for the rate of change of the luminosity of a black hole with temperature $T_{H}$.

At present a black hole will explode if $T_{H}>2.7 \mathrm{~K}$ and correspondingly $M<4.6 \times 10^{25}$ $\mathrm{g}$ which is approximately $1 \%$ of the mass of the Earth. More massive black holes are cooler and therefore will absorb more matter and radiation than they radiate, hence grow with time. Taking into account emission of gravitons, photons, and neutrinos a critical mass black hole today has a Schwarszchild radius of 68 microns and a lifetime of $2 \times 10^{43}$ years. 


\section{Relativistic Viscous Fluid Equations}

The relativistic imperfect fluid equations describing a steady-state, spherically symmetric flow with no net baryon number or electric charge and neglecting gravity (see below) are $T_{; \nu}^{\mu \nu}=$ black hole source. The nonvanishing components of the energy-momentum tensor in radial coordinates are [12]

$$
\begin{aligned}
& T^{00}=\gamma^{2}(P+\epsilon)-P+v^{2} \Delta T_{\text {diss }} \\
& T^{0 r}=v \gamma^{2}(P+\epsilon)+v \Delta T_{\text {diss }} \\
& T^{r r}=v^{2} \gamma^{2}(P+\epsilon)+P+\Delta T_{\text {diss }}
\end{aligned}
$$

representing energy density, radial energy flux, and radial momentum flux, respectively, in the rest frame of the black hole. Here $v$ is the radial velocity with $\gamma$ the corresponding Lorentz factor, $u=v \gamma, \epsilon$ and $P$ are the local energy density and pressure, and

$$
\Delta T_{\mathrm{diss}}=-\frac{4}{3} \eta \gamma^{2}\left(\frac{d u}{d r}-\frac{u}{r}\right)-\zeta \gamma^{2}\left(\frac{d u}{d r}+\frac{2 u}{r}\right)
$$

where $\eta$ is the shear viscosity and $\zeta$ is the bulk viscosity. A thermodynamic identity gives $T s=P+\epsilon$ for zero chemical potentials, where $T$ is temperature and $s$ is entropy density. There are two independent differential equations of motion to solve for the functions $T(r)$ and $v(r)$. These may succinctly written as

$$
\begin{aligned}
& \frac{d}{d r}\left(r^{2} T^{0 r}\right)=0 \\
& \frac{d}{d r}\left(r^{2} T^{r r}\right)=0 .
\end{aligned}
$$

An integral form of these equations is sometimes more useful since it can readily incorporate the input luminosity from the black hole. The first represents the equality of the energy flux passing through a sphere of radius $r$ with the luminosity of the black hole.

$$
4 \pi r^{2} T^{0 r}=L
$$

The second follows from integrating a linear combination of the differential equations. It represents the combined effects of the entropy from the black hole together with the increase of entropy due to viscosity.

$$
4 \pi r^{2} u s=4 \pi \int_{r_{0}}^{r} d r^{\prime} r^{\prime 2} \frac{1}{T}\left[\frac{8}{9} \eta\left(\frac{d u}{d r^{\prime}}-\frac{u}{r^{\prime}}\right)^{2}+\zeta\left(\frac{d u}{d r^{\prime}}+\frac{2 u}{r^{\prime}}\right)^{2}\right]+\frac{L}{T_{H}}
$$

The term $L / T_{H}$ arises from equating the entropy per unit time lost by the black hole $-d S_{\mathrm{bh}} / d t$ with that flowing into the matter. Using the area formula for the entropy of a 
black hole, $S_{\mathrm{bh}}=m_{\mathrm{P}}^{2} \pi r_{S}^{2}=4 \pi M^{2} / m_{\mathrm{P}}^{2}$, and identifying $-d M / d t$ with the luminosity, the entropy input from the black hole is obtained.

The above pair of equations are to be applied beginning at some radius $r_{0}$ greater than the Schwarzschild radius $r_{S}$, that is, outside the quantum particle production region of the black hole. The radius $r_{0}$ at which the imperfect fluid equations are first applied should be chosen to be greater than the Schwarzschild radius, otherwise the computation of particle creation by the black hole would be invalid. It should not be too much greater, otherwise particle collisions would create more entropy than is accounted for by the equation above. The energy and entropy flux into the fluid come from quantum particle creation by the black hole at temperature $T_{H}$. Gravitational effects are of order $r_{S} / r$, hence negligible for $r>(5-10) r_{S}$.

\section{Equation of State and Transport Coefficients}

Determination of the equation of state as well as the two viscosities for temperatures ranging from $\mathrm{MeV}$ to $\mathrm{TeV}$ and more is a formidable task. Here we shall present some relatively simple parametrizations that seem to contain the essential physics. Improvements to these can certainly be made, but probably won't change the viscous fluid flow or the observational consequences very much.

The hot shell of matter surrounding a primordial black hole provides a theoretical testing ground rivaled only by the big bang itself. To illustrate this we have plotted a semi-realistic parametrization of the equation of state in figure 1. Gravitons and neutrinos are not included. We assume (for fun) a second order electroweak phase transition at a temperature of $T_{E W}=100 \mathrm{GeV}$. Above that temperature the standard model has 101.5 effective massless bosonic degrees of freedom (as usual fermions count as $7 / 8$ of a boson). We assume (also for fun) a first order QCD phase transition at a temperature of $T_{Q C D}$ $=170 \mathrm{MeV}$. The number of effective massless bosonic degrees of freedom changes from 47.5 just above this critical temperature ( $\mathrm{u}, \mathrm{d}, \mathrm{s}$ quarks and gluons) to 7.5 just below it (representing the effects of all the massive hadrons in the particle data tables) [13]. Below $30 \mathrm{MeV}$ only electrons, positrons, and photons remain, and finally below a few hundred $\mathrm{keV}$ only photons survive in any appreciable number. The explicit parametrization shown in figure 1 is as follows.

$$
s(T)=\frac{4 \pi^{2}}{90} T^{3} \begin{cases}101.5 & T_{E W} \leq T \\ 56.5+45 \mathrm{e}^{-\left(T_{E W}-T\right) / T} & T_{Q C D} \leq T<T_{E W} \\ 2+3.5 \mathrm{e}^{-m_{e} / T}+27.25 \mathrm{e}^{-\left(T_{Q C D}-T\right) / T} & T<T_{Q C D}\end{cases}
$$

A word about neutrinos: It is quite possible that they should be considered in approximate equilibrium at temperatures above $100 \mathrm{GeV}$ where the electroweak symmetry is restored. Still there is some uncertainty about this. Since they provide only a few effective degrees of freedom out of more than 100 their neglect should cause negligible error. We will address neutrinos and their emission in a subsequent paper. 
Now we turn to the viscosities. The shear viscosity was calculated in 14 for the full standard model in the symmetry restored phase, meaning temperatures above $100 \mathrm{GeV}$ or so, using the relaxation time approximation. The result is

$$
\eta(T>100 \mathrm{GeV})=82.5 T^{3}
$$

when numerical values for coupling constants etc. are put in. The shear viscosity for QCD degrees of freedom only was calculated to leading order in the QCD coupling $\alpha_{s}$ in [15] to be

$$
\eta(\mathrm{QCD})=\frac{0.342\left(1+1.7 N_{f}\right)}{\left(1+N_{f} / 6\right) \alpha_{s}^{2} \ln \left(\alpha_{s}^{-1}\right)} T^{3}
$$

where $N_{f}$ is the number of quark flavors whose mass is less than $T$. This is the extent of our knowledge of shear viscosity at high temperature. We observe that the ratio of the shear viscosity to the entropy density, as appropriate for the above two cases, is dimensionless and has about the same numerical value in both. Therefore, as a practical matter we assume that the shear viscosity always scales with the entropy density for all temperatures of interest. We take the constant of proportionality from the full standard model cited above.

$$
\eta=\frac{82.5}{101.5}\left(\frac{s}{4 \pi^{2} / 90}\right)
$$

There is even less known about the bulk viscosity at the temperatures of interest to us. The bulk viscosity is zero for point particles with no internal degrees of freedom and with local interactions among them. In renormalizable quantum field theories the interactions are not strictly local. In particular, the coupling constants acquire temperature dependence according to the renormalization group. For example, to one loop order the QCD coupling has the functional dependence $\alpha_{s} \sim 1 / \ln (T / \Lambda)$ where $\Lambda$ is the QCD scale. On account of this dependence the bulk viscosity is nonzero. We estimate that

$$
\zeta \approx 10^{-4} \eta
$$

and this is what we shall use in the numerics.

Overall we have a modestly realistic description of the equation of state and the viscosities that are still a matter of theoretical uncertainty. One needs $s(T), \eta(T), \zeta(T)$ over a huge range of $T$. Of course, these are some of the quantities one hopes to obtain experimental information on from observations of exploding black holes.

\section{$5 \quad$ Numerical Solution and Scaling}

Several limiting cases of the relativistic viscous fluid equations were studied in [9]. The most realistic situation used the equation of state $\epsilon=a T^{4}, s=(4 / 3) a T^{3}$ and viscosities $\eta=b_{S} T^{3}, \zeta=b_{B} T^{3}$ with the coefficients $a, b_{S}, b_{B}$ all constant. A scaling solution, valid 
at large radii when $\gamma \gg 1$, was found to be $T(r)=T_{0}\left(r_{0} / r\right)^{2 / 3}$ and $\gamma(r)=\gamma_{0}\left(r / r_{0}\right)^{1 / 3}$. The constants must be related by $36 a T_{0} r_{0}=\left(32 b_{S}+441 b_{B}\right) \gamma_{0}$. This $r$-dependence of $T$ and $\gamma$ is exactly what was conjectured by Heckler [0].

It was shown in [9] that if approximate local thermal equilibrium is achieved it can be maintained, at least for the semi-realistic situation described above. The requirement is that the inverse of the local volume expansion rate $\theta=u_{; \mu}^{\mu}$ be comparable to or greater than the relaxation time for thermal equilibrium [12]. Expressed in terms of a local volume element $V$ and proper time $\tau$ it is $\theta=(1 / V) d V / d \tau$, whereas in the rest frame of the black hole the same quantity can be expressed as $\left(1 / r^{2}\right) d\left(r^{2} u\right) / d r$. Explicitly

$$
\theta=\frac{7 \gamma_{0}}{3 r_{0}}\left(\frac{r_{0}}{r}\right)^{2 / 3}=\frac{7 \gamma_{0}}{3 r_{0} T_{0}} T .
$$

Of prime importance in achieving and maintaining local thermal equilibrium in a relativistic plasma are multi-body processes such as $2 \rightarrow 3$ and $3 \rightarrow 2$, etc. This has been well-known when calculating quark-gluon plasma formation and evolution in high energy heavy ion collisions [16, 17] and has been emphasized in ref. [6, 7] in the context of black hole evaporation. This is a formidable task in the standard model with its 16 species of particles. Instead three estimates for the requirement that local thermal equilibrium be maintained were made. The first and simplest estimate is to require that the thermal DeBroglie wavelength of a massless particle, $1 / 3 T$, be less than $1 / \theta$. The second estimate is to require that the Debye screening length for each of the gauge groups in the standard model be less than $1 / \theta$. The Debye screening length is the inverse of the Debye screening mass $m_{n}^{\mathrm{D}}$ where $n=1,2,3$ for the gauge groups $\mathrm{U}(1), \mathrm{SU}(2)$, SU(3). Generically $m_{n}^{\mathrm{D}} \propto g_{n} T$ where $g_{n}$ is the gauge coupling constant and the coefficient of proportionality is essentially the square root of the number of charge carriers $18 \|$. For example, for color $\mathrm{SU}(3) m_{3}^{\mathrm{D}}=g_{3} \sqrt{1+N_{\mathrm{f}} / 6} T$ where $N_{\mathrm{f}}$ is the number of light quark flavors at the temperature $T$. The numerical values of the gauge couplings are: $g_{1}=0.344, g_{2}=0.637$, and $g_{3}=1.18$ (evaluated at the scale $m_{Z}$ ) [3]. So within a factor of about 2 we have $m^{\mathrm{D}} \approx T$. The third and most relevant estimate is the mean time between two-body collisions in the standard model for temperatures greater than the electroweak symmetry restoration temperature. This mean time was calculated in [14] in the process of calculating the viscosity in the relaxation time approximation. Averaged over all particle species in the standard model one may infer from that paper an average time of $3.7 / T$. Taking into account multi-body reactions would decrease that by about a factor of two to four. All three of these estimates are consistent within a factor of 2 or 3 . The conclusion to be drawn is that local thermal equilibrium should be achieved when $\theta \lesssim T$. Once thermal equilibrium is achieved it is not lost because $\theta / T$ is independent of $r$. The picture that emerges is that of an imperfect fluid just marginally kept in local equilibrium by viscous forces.

The results quoted above are only valid at large $r$ and for the equation of state $s \propto T^{3}$. To know the behavior of the solution at non-asymptotic $r$ and for the more sophisticated 
equation of state and viscosities described in section 5 requires a numerical analysis. We have found that the most convenient form of the viscous fluid equations for numerical evaluation are

$$
4 \pi r^{2}\left[\gamma u T s-\frac{4}{3} \eta \gamma u\left(\frac{d u}{d r}-\frac{u}{r}\right)-\zeta \gamma u\left(\frac{d u}{d r}+\frac{2 u}{r}\right)\right]=L
$$

for energy conservation (from eq. (9)) and

$$
\frac{d}{d r}\left(4 \pi r^{2} u s\right)=\frac{4 \pi r^{2}}{T}\left[\frac{8}{9} \eta\left(\frac{d u}{d r}-\frac{u}{r}\right)^{2}+\zeta\left(\frac{d u}{d r}+\frac{2 u}{r}\right)^{2}\right]
$$

for entropy flow (from eq. (10)). Obviously the entropy flux is a monotonically increasing function of $r$ because of dissipation.

Mathematically the above pair of equations apply for all $r>0$, although physically we should only apply them beyond the Schwarzschild radius $r_{S}$. Let us study them first in the limit $r \rightarrow 0$, which really means the assumption that $v \ll 1$. Then $u \approx v$ and $\gamma \approx 1$. We also consider black hole temperatures greater than $T_{E W}$ so that the equation of state and the viscosities no longer change their functional forms. It is straightforward to check that a power solution satisfies the equations, with

$$
\begin{aligned}
u(r) & =u_{i}\left(r / r_{i}\right)^{2 / 5} \\
T(r) & =T_{i}\left(r_{i} / r\right)^{3 / 5}
\end{aligned}
$$

where $r_{i}$ is some reference radius. If the luminosity and the reference radius are given then $u_{i}$ and $T_{i}$ are determined by the fluid equations.

The numerical solution for all radii needs some initial conditions. Typically we begin the solution at one-tenth the Schwarzschild radius. At this radius the $u_{i}$, as determined above, is small enough to serve as a good first estimate. However, it needs to be fine-tuned to give an acceptable solution at large $r$. For example, at large $r$ there is an approximate but false solution: $T=$ constant with $u \sim r$. The problem is that we need a solution valid over many orders of magnitude of $r$. If eq. (17) is divided by $r^{2}$ and if the right hand side is neglected in the limit $r \rightarrow \infty$ then the left hand side is forced to be zero. We have used both Mathematica and a fourth order Runge-Kutta method with adaptive step-size to solve the equations. They give consistent results.

In figure 2 we plot $u\left(r_{S} / 10\right)$ versus $T_{H}$. It is essentially constant for all $T_{H}>T_{E W}$ with the value of 0.0415 . In figure 3 we plot the function $u(r)$ versus $r$ for three different black hole temperatures. The radial variable $r$ is expressed in units of its value when the temperature first reaches $T_{Q C D}$, and $u$ is expressed in units of its value at that same radius. This allows us to compare different black hole temperatures. To rather good accuracy these curves seem to be universal as they essentially lie on top of one another. The curves 
are terminated when the temperature reaches $10 \mathrm{MeV}$. The function $u(r)$ behaves like $r^{1 / 3}$ until temperatures of order $100 \mathrm{MeV}$ are reached. The simple parametrization

$$
u(r)=u_{S}\left(r / r_{S}\right)^{1 / 3}
$$

with $u_{S}=0.10$ will be very useful when studying radiation from the surface of the fluid.

In figure 4 we plot the temperature in units of $T_{Q C D}$ versus the radius in units of $r_{Q C D}$ for the same three black hole temperatures as in figure 3. Again the curves are terminated when the temperature drops to $10 \mathrm{MeV}$. The curves almost fall on top of one another but not perfectly. The temperature falls slightly slower than the power-law behavior $r^{-2 / 3}$ expected on the basis of the equation of state $s=(4 / 3) a T^{3}$. The reason is that the effective number of degrees of freedom is falling with the temperature. The entropy density is shown in figure 5. It also exhibits an imperfect degree of scaling similar to the temperature.

Since viscosity plays such an important role in the outgoing fluid we should expect significant entropy production. In figure 6 we plot the entropy flow $4 \pi r^{2} u s$ as a function of radius for the same three black hole temperatures as in figures 3-5. It increases by several orders of magnitude. The fluid flow is far from isentropic.

\section{Onset of Free-Streaming}

Eventually the fluid expands so rapidly that the particles composing the fluid lose thermal contact with each other and begin free-streaming. In heavy ion physics this is referred to as thermal freeze-out, and in astronomy it is usually associated with the photosphere of a star. In the sections above we argued that thermal contact should occur for all particles, with the exception of gravitons and neutrinos, down to temperatures on the order of $T_{Q C D}$. Below that temperature the arguments given no longer apply directly; for example, the relevant interactions are not those of perturbative QCD.

Extensive studies have been made of the interactions among hadrons at finite temperature. Prakash et al. [19 used experimental information to construct scattering amplitudes for pions, kaons and nucleons and from them computed thermal relaxation rates. The relaxation time for $\pi-\pi$ scattering can be read off from their figures and simply paramterized as

$$
\tau_{\pi \pi}^{-1} \approx 16\left(\frac{T}{100 \mathrm{MeV}}\right)^{4} \mathrm{MeV}
$$

which is valid for $100<T<200 \mathrm{MeV}$. This rate is compared to the volume expansion rate $\theta$ (see section 5 ) in figure 7 . From the figure it is clear that pions cannot maintain thermal equilibrium much below $160 \mathrm{MeV}$ or so. Since pions are the lightest hadrons and therefore the most abundant at low temperatures, it seems unlikely that other hadrons could maintain thermal equilibrium either. 
Heckler has argued vigorously that electrons and photons should continue to interact

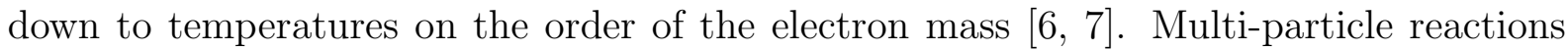
are crucial to this analysis. Let us see how it applies to the present situation. Consider, for example, the cross section for $e e \rightarrow e e \gamma$. The energy-averaged cross section is [7]

$$
\bar{\sigma}_{\text {brem }}=8 \alpha_{E M} r_{0}^{2} \ln \left(2 E / m_{e}\right)
$$

where $m_{e}$ is the electron mass, $r_{0}=\alpha_{E M} / m_{e}$ is the classical electron radius, and $E \gg m_{e}$ is the energy of the incoming electrons in the center-of-momentum frame. (If one computes the rate for a photon produced with the specific energies $0.1 E, 0.25 E$, or $0.5 E$ the cross section would be larger by a factor $4.73,2.63$, or 1.27 , respectively.) The rate using the energy-averaged cross section is

$$
\tau_{\text {brem }}^{-1} \approx\left[\frac{3}{\pi^{2}} \xi(3) T^{3}\right]\left[\frac{8 \alpha_{E M}^{3}}{m_{e}^{2}} \ln \left(6 T / m_{e}\right)\right]
$$

where we have used the average energy $\langle E\rangle \approx 3 T$ for electrons with $m_{e} \ll T$. This rate is also plotted in figure 7 . It is large enough to maintain local thermal equilibrium down to temperatures on the order of $140 \mathrm{MeV}$. Of course, there are other electromagnetic manyparticle reactions which would increase the overall rate. On the other hand, as pointed out by Heckler [7], these reactions are occurring in a high density plasma with the consequence that dispersion relations and interactions are renormalized by the medium. If one takes into account only renormalization of the electron mass, such that $m_{e f f}^{2} \approx m_{e}^{2}+e^{2} T^{2} / 3$ when $m_{e} \ll T$, then the rate would be greatly reduced.

Does this mean that photons and electrons are not in thermal equilibrium at the temperatures we have been discussing? Consider bremsstrahlung reactions in the QCD plasma. There are many $2 \rightarrow 3$ reactions, such as: $q_{1} q_{2} \rightarrow q_{1} q_{2} \gamma, q_{1} \bar{q}_{2} \rightarrow q_{1} \bar{q}_{2} \gamma, g g \rightarrow q \bar{q} \gamma$, and so on. Here the subscripts label the quark flavor, which may or may not be the same. The rate for these can be estimated using known QED and QCD cross sections 20, 21, 22]. Using an effective quark mass given by $g T$ we find that the rate is $\alpha_{s} T$ with a coefficient of order or larger than unity. Since $\alpha_{s}$ becomes of order unity near $T_{Q C D}$ we conclude that photons are in equilibrium down to temperatures of that order at least. To make the matter even more complicated we must remember that the expansion rate $\theta$ is based on a numerical solution of the viscous fluid equations which assume a constant proportionality between the shear and bulk viscosities and the entropy density. Although these proportionalities may be reasonable in QCD and electroweak plasmas at high temperatures they may fail at temperatures below $T_{Q C D}$. The viscosities should be computed using the relaxation times for self-consistency of the transition from viscous fluid flow to free-streaming, which we have not done. For example, the first estimate for the shear viscosity for massless particles with short range interactions is $T^{4} \tau$ where $\tau$ is the relaxation time. For pions we would get $\eta \sim$ constant, not $\eta \sim T^{3}$. As another example, we must realize that the bulk viscosity can become significant when the particles 
can be excited internally. This is, in fact, the case for hadrons. Pions, kaons and nucleons are all the lowest mass hadrons each of which sits at the base of a tower of resonances [3]. See, for example, 23] and references therein.

In order to do gamma ray phenomenology we need a practical criterion for the onset of free-streaming. We shall assume that this happens suddenly at a temperature $T_{f}$ in the range 100 to $140 \mathrm{MeV}$. We shall assume that particles whose mass is significantly greater than $T_{f}$ have all annihilated, leaving only photons, electrons, muons and pions. In figure 8 we plot the freeze-out radius $r_{f}=r\left(T_{f}\right)$ for $T_{f}=100,120$ and $140 \mathrm{MeV}$ versus the Schwarzschild radius. The fact that $r_{f}$ increases as $T_{f}$ decreases is an obvious consequence of energy conservation. More interesting is the power-law scaling: $r_{f} \sim r_{S}^{-1 / 2} \sim T_{H}^{1 / 2}$. This scaling can be understood as follows.

The luminosity from the decoupling or freeze-out surface is

$$
L_{f}=4 \pi r_{f}^{2}\left(\frac{2 \pi^{2}}{45} \gamma_{f}^{2} T_{f}^{4}\right) d_{f}
$$

where the quantity in parentheses is the surface flux for one massless bosonic degree of freedom and $d_{f}$ is the total number of effective massless bosonic degrees of freedom. For the particles listed above we have $d_{f}=12$. By energy conservation this is to be equated with the Hawking formula for the black hole luminosity,

$$
L_{h}=64 \pi^{2} \alpha_{h}^{e f f} T_{H}^{2},
$$

where $\alpha_{h}^{e f f}$ does not include the contribution from gravitons and neutrinos. Together with the scaling function for the flow velocity, eq. (20), we can solve for the radius

$$
r_{f}=\frac{2}{\pi}\left(\frac{45 \pi \alpha_{h}^{e f f}}{2 u_{S}^{2} d_{f}}\right)^{3 / 8} \sqrt{\frac{T_{H}}{T_{f}^{3}}}
$$

and for the boost

$$
\gamma_{f} T_{f}=2 u_{S}\left(\frac{45 \pi \alpha_{h}^{e f f}}{2 u_{S}^{2} d_{f}}\right)^{1 / 8} \sqrt{T_{f} T_{H}} \approx 0.22 \sqrt{T_{f} T_{H}} .
$$

From these we see that the final radius does indeed scale like the inverse of the square-root of the Schwarzschild radius or like the square-root of the black hole temperature, and that the average particle energy (proportional to $\gamma_{f} T_{f}$ ) scales like the square-root of the black hole temperature. One important observational effect is that the average energy of the outgoing particles is reduced but their number is increased compared to direct Hawking emission into vacuum [6, 7]. 


\section{Photon Emission}

Photons emitted into the vacuum surrounding the black hole primarily come from one of two sources. Either they are emitted directly in the form of a boosted black-body spectrum, or they arise from neutral pion decay. We will consider each of these in turn.

\subsection{Direct photons}

Photons emitted directly have a Planck distribution in the local rest frame of the fluid. The phase space density is

$$
f\left(E^{\prime}\right)=\frac{1}{\mathrm{e}^{E^{\prime} / T_{f}}-1} .
$$

The energy appearing here is related to the energy as measured in the rest frame of the black hole and to the angle of emission relative to the radial vector by

$$
E^{\prime}=\gamma_{f}\left(1-v_{f} \cos \theta\right) E .
$$

No photons will emerge if the angle is greater than $\pi / 2$. Therefore the instantaneous distribution is

$$
\begin{aligned}
\frac{d^{2} N_{\gamma}^{\text {dir }}}{d E d t} & =4 \pi r_{f}^{2}\left(\frac{E^{2}}{2 \pi^{2}}\right) \int_{0}^{1} d(\cos \theta) \cos \theta f(E, \cos \theta) \\
& \approx-\frac{2 r_{f}^{2} T_{f} E}{\pi \gamma_{f}} \ln \left(1-\mathrm{e}^{-E / 2 \gamma_{f} T_{f}}\right)
\end{aligned}
$$

where the second equality holds in the limit $\gamma_{f} \gg 1$. This limit is actually well satisfied for us and is used henceforth.

The instantaneous spectrum can be integrated over the remaining lifetime of the black hole straightforwardly. The radius and boost are both known in terms of the Hawking temperature $T_{H}$, and the time evolution of the latter is simply obtained from solving eq.(3). For a black hole that disappears at time $t=0$ we have

$$
T_{H}(t)=-\frac{1}{8 \pi}\left(\frac{m_{P}^{2}}{3 \alpha_{h} t}\right)^{1 / 3}
$$

Starting with a black hole whose temperature is $T_{0}$ we obtain the spectrum

$$
\frac{d N_{\gamma}^{\text {dir }}}{d E}=\frac{360 u_{S}^{2}}{\pi^{5} d_{f}}\left(\frac{45 \pi \alpha_{h}^{e f f}}{2 u_{S}^{2} d_{f}}\right)^{1 / 4} \frac{m_{P}^{2} T_{f}}{E^{4}} \sum_{n=1}^{\infty} \frac{1}{n} \int_{0}^{E / 2 \gamma_{f}\left(T_{0}\right) T_{f}} d x x^{4} \mathrm{e}^{-n x}
$$

Here we have ignored the small numerical difference between $\alpha_{h}^{e f f}$ and $\alpha_{h}$. In the high energy limit, namely, when $E \gg 2 \gamma_{f}\left(T_{0}\right) T_{f}$, the summation yields the pure number $4\left(2 \pi^{6} / 315\right)$. Note the power-law behavior $E^{-4}$. This has important observational consequences. 


\section{$7.2 \quad \pi^{0}$ decay photons}

The neutral pion decays almost entirely into two photons: $\pi^{0} \rightarrow \gamma \gamma$. In the rest frame of the pion the single photon Lorentz invariant distribution is

$$
E \frac{d^{3} N_{\gamma}}{d^{3} p}=\frac{\delta\left(E-m_{\pi} / e\right)}{\pi m_{\pi}}
$$

which is normalized to 2. This must be folded with the distribution of $\pi^{0}$ to obtain the total invariant photon distribution.

$$
E \frac{d^{4} N_{\gamma}}{d^{3} p d t}=\int_{m_{\pi}}^{\infty} d E_{\pi} \frac{d^{2} N_{\pi^{0}}}{d E_{\pi} d t} \frac{1}{\pi m_{\pi}} \delta\left(\frac{E E_{\pi}-\mathbf{p} \cdot \mathbf{p}_{\pi}}{m_{\pi}}-\frac{m_{\pi}}{2}\right)
$$

After integrating over angles we get

$$
\frac{d^{2} N_{\gamma}^{\pi^{0}}}{d E d t}=2 \int_{E_{\min }}^{\infty} \frac{d E_{\pi}}{p_{\pi}} \frac{d^{2} N_{\pi^{0}}}{d E_{\pi} d t}
$$

where $E_{\min }=\left(E^{2}+m_{\pi}^{2} / 4\right) / E$. In the limit $E \gg m_{\pi}$ we can approximate $E_{\min }=E$ and evaluate $d^{2} N_{\pi^{0}} / d E_{\pi} d t$ in the same way as photons. This leads to the relatively simple expression

$$
\frac{d^{2} N_{\gamma}^{\pi^{0}}}{d E d t}=\frac{4 r_{f}^{2} T_{f}^{2}}{\pi^{2}} \sum_{n=1}^{\infty} \frac{1}{n^{2}} \mathrm{e}^{-n E / 2 \gamma_{f} T_{f}}
$$

The time-integrated spectrum is computed in the same way as for direct photons.

$$
\frac{d N_{\gamma}^{\pi^{0}}}{d E}=\frac{360 u_{S}^{2}}{\pi^{5} d_{f}}\left(\frac{45 \pi \alpha_{h}^{e f f}}{2 u_{S}^{2} d_{f}}\right)^{1 / 4} \frac{m_{P}^{2} T_{f}}{E^{4}} \sum_{n=1}^{\infty} \frac{1}{n^{2}} \int_{0}^{E / 2 \gamma_{f}\left(T_{0}\right) T_{f}} d x x^{3} \mathrm{e}^{-n x}
$$

In the high energy limit, namely, when $E \gg 2 \gamma_{f}\left(T_{0}\right) T_{f}$, the summation yields the pure number $2 \pi^{6} / 315$.

\subsection{Instantaneous and integrated photon spectra}

The instantaneous spectra of high energy gamma rays, arising from both direct emission and from $\pi^{0}$ decay, are plotted in figures 9 (for $T_{f}=140 \mathrm{MeV}$ ) and 10 (for $T_{f}=100$ $\mathrm{MeV})$. In each figure there are three curves corresponding to Hawking temperatures of $100 \mathrm{GeV}, 1 \mathrm{TeV}$ and $10 \mathrm{TeV}$. The photon spectra are essentially exponential above a few $\mathrm{GeV}$ with inverse slope $2 \gamma_{f}\left(T_{H}\right) T_{f} \propto \sqrt{T_{f} T_{H}}$. If these instantaneous spectra could be measured they would tell us a lot about the equation of state, the viscosities, and how energy is processed from first Hawking radiation to final observed gamma rays. Even the time evolution of the black hole luminosity and temperature could be inferred. 
The time integrated spectra for $T_{f}=140 \mathrm{MeV}$ are plotted in figure 11 for three initial temperatures $T_{0}$. A black hole with a Hawking temperature of $100 \mathrm{GeV}$ has 5.4 days to live, a black hole with a Hawking temperature of $1 \mathrm{TeV}$ has 7.7 minutes to live, and a black hole with a Hawking temperature of $10 \mathrm{TeV}$ has only $1 / 2$ second to live. The high energy gamma ray spectra are represented by

$$
\frac{d N}{d E}=\frac{m_{P}^{2} T_{f}}{26 E^{4}}
$$

It is interesting that the contribution from $\pi^{0}$ decay comprises $20 \%$ of the total while direct photons contribute the remaining $80 \%$. The $E^{-4}$ fall-off is the same as that obtained by Heckler [6], whereas Halzen et al. [24] and MacGibbon and Carr [5] obtained an $E^{-3}$ fall-off on the basis of direct fragmentation of quarks and gluons with no fluid flow and no photosphere.

\section{Observability of Gamma Rays}

The most obvious way to observe the explosion of a microscopic black hole is by high energy gamma rays. We consider their contribution to the diffuse gamma ray spectrum in subsection 8.1, and in subsection 8.2 we study the systematics of a single identifiable explosion.

\subsection{Diffuse spectra from the galactic halo}

Suppose that microscopic black holes were distributed about our galaxy in some fashion. Unless we were fortunate enough to be close to one so that we could observe its demise, we would have to rely on their contribution to the diffuse background spectrum of high energy gamma rays.

The flux of photons with energy greater than $1 \mathrm{GeV}$ at Earth can be computed from the results of section 7 together with the knowledge of the rate density $\dot{\rho}(\mathbf{x})$ of exploding black holes. It is

$$
\frac{d^{3} N_{\mathrm{Earth}}}{d E d A d t}=\frac{m_{P}^{2} T_{f}}{26 E^{4}} \int d^{3} x \frac{\dot{\rho}(\mathbf{x})}{4 \pi d^{2}(\mathbf{x})} \mathrm{e}^{-d(\mathbf{x}) / \lambda_{\gamma \gamma}(E)}
$$

where $d(\mathbf{x})$ is the distance from the black hole to the Earth. The exponential decay is due to absorption of the gamma ray by the black-body radiation [25]. The mean free path $\lambda_{\gamma \gamma}(E)$ is highly energy dependent. It has a minimum of about $1 \mathrm{kpc}$ around $1 \mathrm{PeV}$, and is greater than $10^{5} \mathrm{kpc}$ for energies less than $100 \mathrm{TeV}$.

We need a model for the rate density of exploding black holes. We shall assume they are distributed in the same way as the matter comprising the halo of our galaxy. Thus we take

$$
\dot{\rho}(\mathbf{x})=\dot{\rho}_{0} \frac{R_{c}^{2}}{x^{2}+y^{2}+q^{2} z^{2}+R_{c}^{2}}
$$


where the galactic plane is the $x-y$ plane, $R_{c}$ is the core radius, and $q$ is a flattening parameter. For numerical calculations we shall take the core radius to be $10 \mathrm{kpc}$. The Earth is located a distance $R_{E}=8.5 \mathrm{kpc}$ from the center of the galaxy and lies in the galactic plane. Therefore $d^{2}=\left(x-R_{E}\right)^{2}+y^{2}+z^{2}$.

The last remaining quantity is the normalization of the rate density $\dot{\rho}_{0}$. This is, of course, unknown since no one has ever knowingly observed a black hole explosion. The first observational limit was determined by Page and Hawking [26]. They found that the local rate density $\dot{\rho}_{\text {local }}$ is less than 1 to 10 per cubic parsec per year on the basis of diffuse gamma rays with energies on the order of $100 \mathrm{MeV}$. This limit has not been lowered very much during the intervening twenty-five years. For example, Wright 27 used EGRET data to search for an anisotropic high-lattitude component of diffuse gamma rays in the energy range from $30 \mathrm{MeV}$ to $100 \mathrm{GeV}$ as a signal for steady emission of microscopic black holes. He concluded that $\dot{\rho}_{\text {local }}$ is less than about 0.4 per cubic parsec per year. (For an alternative point of view on the data see 28].) In our numerical calculations we shall assume a value $\dot{\rho}_{0}=1 \mathrm{pc}^{-3} \mathrm{yr}^{-1}$ corresponding to $\dot{\rho}_{\text {local }} \approx 0.58 \mathrm{pc}^{-3} \mathrm{yr}^{-1}$. This makes for easy scaling. Estimating the quantity of dark matter in our galaxy as $M_{\text {halo }} / \rho_{0 \text {, halo }}=4.7 \times 10^{4} \mathrm{kpc}^{3}$ means that we could have up to $47 \times 10^{12}$ microscopic black hole explosions per year in our galaxy.

Figure 12 shows the calculated flux at Earth, multiplied by $E^{4}$. Of course this curve would be flat if it weren't for absorption on the microwave background radiation. There is a relative suppression of three orders of magnitude centered between $10^{15}$ and $10^{16} \mathrm{eV}$. This means that it is unlikely to observe exploding black holes in the gamma ray spectrum above $10^{14} \mathrm{eV}$. Even below that energy it is unlikely because they have not been observed at energies on the order of $100 \mathrm{MeV}$, and the spectrum falls faster than the primary cosmic ray spectrum $\propto E^{2.7}$. The curve displayed in figure 12 assumes a spherical halo, $q=1$, but there is hardly any difference when the halo is flattened to $q=2$.

\subsection{Point source systematics}

Given the unfavorable situation for observing the effects of exploding microscopic black holes on the diffuse gamma ray spectrum, we now turn to the consequences for observing one directly. How far away could one be seen? Let us call that distance $d_{\max }$. We assume that $d_{\max }<\lambda_{\gamma \gamma}$ for simplicity, although that assumption can be relaxed if necessary. Let $A_{\text {det }}$ denote the effective area of the detector that can measure gamma rays with energies equal to or greater than $E_{\text {min }}$. The average number of gamma rays detected from a single explosion a distance $d_{\max }$ away is

$$
\left\langle N_{\gamma}\left(E>E_{\min }\right)\right\rangle=\frac{A_{\mathrm{det}}}{4 \pi d_{\max }^{2}} \int_{E_{\min }}^{\infty} \frac{d N_{\gamma}}{d E} d E=\frac{A_{\mathrm{det}}}{4 \pi d_{\max }^{2}} \frac{m_{P}^{2} T_{f}}{78 E_{\min }^{3}} .
$$

Obviously we should have $E_{\min }$ as small as possible to get the largest number, but it cannot be so small that the simple $E^{-4}$ behavior of the emission spectrum is invalid. See 
figure 11.

A rough approximation to the number distribution of detected gamma rays is a Poisson distribution.

$$
P\left(N_{\gamma}\right)=\frac{\left\langle N_{\gamma}\right\rangle^{N_{\gamma}}}{N_{\gamma} !} \mathrm{e}^{-\left\langle N_{\gamma}\right\rangle}
$$

The exact form of the number distribution is not so important. What is important is that when $\left\langle N_{\gamma}\left(E>E_{\text {min }}\right)\right\rangle>1$ we should expect to see multiple gamma rays coming from the same point in the sky. Labeling these gamma rays according to the order in which they arrive, 1, 2, 3, etc. we would expect their energies to increase with time: $E_{1}<E_{2}<E_{3}<\ldots$. Such an observation would be remarkable, possibly unique, because astrophysical sources normally cool at late times. This would directly reflect the increasing Hawking temperature as the black hole explodes and disappears.

It is interesting to know how the average gamma ray energy increases with time. Using eqs. (30) and (36) we compute the average energy of direct photons to be $4 \gamma_{f} T_{f} \zeta(4) / \zeta(3)$ and the average energy of $\pi^{0}$ decay photons to be one-half that. The ratio of direct to decay photons turns out to be $\pi$. Therefore the average gamma ray energy is $3.17 \gamma_{f}(t) T_{f}$. This average is plotted in figure 13 for $10^{5}>t>10^{-5}$ seconds. The average gamma ray energy ranges from about 4 to $160 \mathrm{GeV}$.

The maximum distance can now be computed. Using some characteristic numbers we find

$$
d_{\max } \approx 150 \sqrt{\frac{A_{\mathrm{det}}}{1 \mathrm{~km}^{2}}}\left(\frac{10 \mathrm{GeV}}{E_{\min }}\right)^{3 / 2} \mathrm{pc} .
$$

If we take the local rate density of explosions to be $0.4 \mathrm{pc}^{-3} \mathrm{yr}^{-1}$ then within $150 \mathrm{pc}$ of Earth there would be $5 \times 10^{6}$ explosions per year. These would be distributed isotropically in the sky. Still, it suggests that the direct observation of exploding black holes is feasible if they are near to the inferred upper limit to their abundance in our neighborhood. We should point out that a search for $1 \mathrm{~s}$ bursts of ultrahigh energy gamma rays from point sources by CYGNUS has placed an upper limit of $8.5 \times 10^{5} \mathrm{pc}^{-3} \mathrm{yr}^{-1}$ [29]. However, as we have seen in figure 13 and elsewhere, this is not what should be expected if our calculations bear any resemblance to reality. Rather than a burst, the luminosity and average gamma ray energy increase monotonically over a long period of time.

\section{Conclusion}

The increasing energy of the radiated photons by an exploding black hole and the disappearance of such a point gamma ray source in a certain period of time are unique characteristics of exploding black holes that may help us to detect them. Still, there is much work to be done in determining whether the matter surrounding a black hole can reach and maintain thermal equilibrium. The equation of state should be improved, and the viscosities computed using the relaxation times for self-consistency of the transition 
from viscous fluid flow to free streaming. Also, there should be a more fundamental investigation of the relaxation times starting from the microscopic interactions.

Our next step is to calculate the neutrino flux radiated by exploding black holes. Neutrino cross sections become very small at energies below $100 \mathrm{Gev}$ which is the temperature of the electroweak phase transition. Above $100 \mathrm{GeV}$ the neutrino cross sections are the same as other particles in the standard model which allows neutrinos to interact enough to reach thermal equilibrium. Therefore neutrinos are expected to freeze out at a temperature around $100 \mathrm{GeV}$. Another worthwhile project is to carry out cascade simulations of the spherically expanding matter around the exploding black hole at a level of sophistication comparable to that of high energy heavy ion collisions. This project is much more complicated than the cascade simulation in heavy ion collision, though, because we need to deal with a much wider range of energies and particles involved in exploding black holes.

The study of primordial black holes might well lead to great advancements in fundamental physics. Because the highest temperatures in the universe exist in primordial black holes, matter at extremely high temperatures can be studied, and physics beyond the standard model can be tested. In addition, because it is believed that baryon number is violated at high temperatures, the study of primordial black holes could possibly answer the question of why our universe became matter-dominated. Because primordial black holes explode, they are an ideal model for studying the Big Bang and the birth of our universe. Finally, the study of primordial black holes will help us to determine whether they are the source of the highest energy cosmic rays. The origin of these cosmic rays is still one of the biggest mysteries today. Observation and experimental detection of exploding black holes will be one of the great challenges in the new millennium.

\section{Acknowledgements}

This work was supported by the US Department of Energy under grant DE-FG0287ER40328.

\section{References}

[1] S. W. Hawking, Nature (London) 248, 30 (1974); Commun. Math. Phys. 43, 199 (1975).

[2] B. J. Carr and J. H. MacGibbon, Phys. Rep. 307, 141 (1998).

[3] Particle Data Group: Review of Particle Physics, Eur. Phys. J. C3, 1 (1998).

[4] J. H. MacGibbon and B. R. Webber, Phys. Rev. D 41, 3052 (1990). 
[5] J. H. MacGibbon and B. J. Carr, Astrophys. J. 371, 447 (1991).

[6] A. F. Heckler, Phys. Rev. Lett. 78, 3430 (1997).

[7] A. F. Heckler, Phys. Rev. D 55, 480 (1997).

[8] J. Cline, M. Mostoslavsky, and G. Servant, Phys. Rev. D 59, 063009 (1999).

[9] J. I. Kapusta, Phys. Rev. Lett. 86, 1670 (2001).

[10] D. N. Page, Phys. Rev. D 13, 198 (1976).

[11] N. Sanchez, Phys. Rev. D 18, 1030 (1978); the coefficient was extracted from figure 5 of this paper to within about $5 \%$ accuracy.

[12] C. W. Misner, K. S. Thorne, and J. A. Wheeler, Gravitation (W. H. Freeman and Company, San Francisco, 1973).

[13] J. I. Kapusta and K. A. Olive, Phys. Lett. B209, 295 (1988).

[14] M. E. Carrington and J. I. Kapusta, Phys. Rev. D 47, 5304 (1993).

[15] G. Baym, H. Monien, C. J. Pethick, and D. G. Ravenhall, Phys. Rev. Lett. 64, 1867 (1990).

[16] K. Kinder-Geiger, Phys. Rep. 258, 237 (1995).

[17] S.M.H. Wong, Phys. Rev. C 54, 2588 (1996).

[18] J. I. Kapusta, Finite Temperature Field Theory (Cambridge University Press, Cambridge, England, 1989).

[19] M. Prakash, M. Prakash, R. Venugopalan and G. Welke, Phys. Rep. 227, 321 (1993).

[20] J. M. Jauch and F. Rohrlich, The Theory of Electrons and Photons, (Springer-Verlag, New York, 1975).

[21] E. Haug, Z. Naturforsch. Teil A 30, 1099 (1975).

[22] R. Cutler and D. Sivers, Phys. Rev. D 17, 196 (1978).

[23] S. Weinberg, Gravitation and Cosmology, (John Wiley \& Sons, New York, 1972), p. 57.

[24] F. Halzen, E. Zas, J. H. MacGibbon and T. C. Weekes, Nature (London) 353, 807 (1991).

[25] R. J. Gould and G. Schreder, Phys. Rev. Lett. 16, 252 (1966). 
[26] D. N. Page and S. W. Hawking, Astrophys. J. 206, 1 (1976).

[27] E. L. Wright, Astrophys. J. 459, 487 (1996).

[28] D. B. Cline, Astrophys. J. 501, L1 (1998).

[29] D. E. Alexandreas, et al., Phys. Rev. Lett. 71, 2524 (1993). 


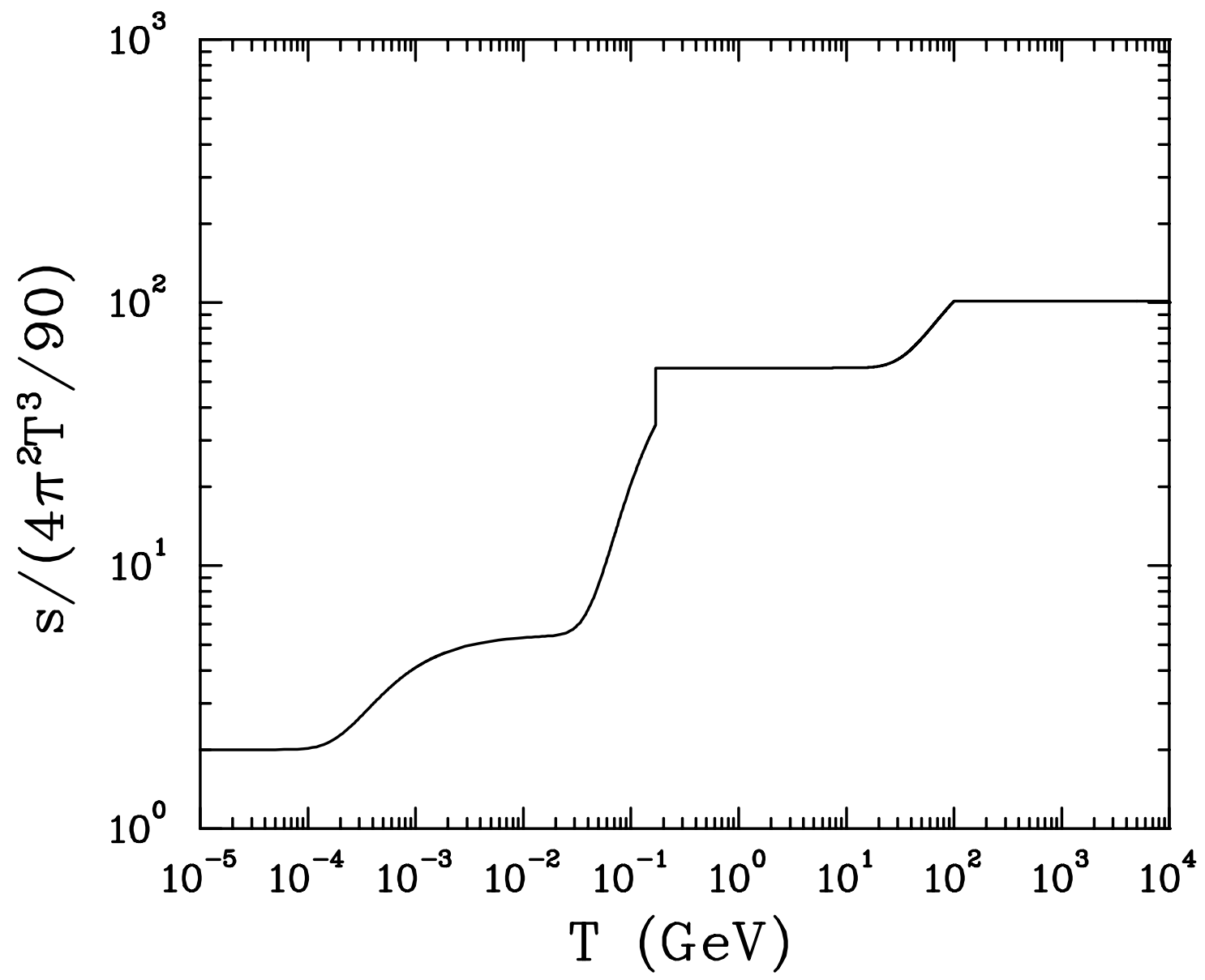

Figure 1: Entropy density as a function of temperature, excluding neutrinos and gravitons. It is assumed that the QCD phase transition is first order and the EW phase transition is second order. 


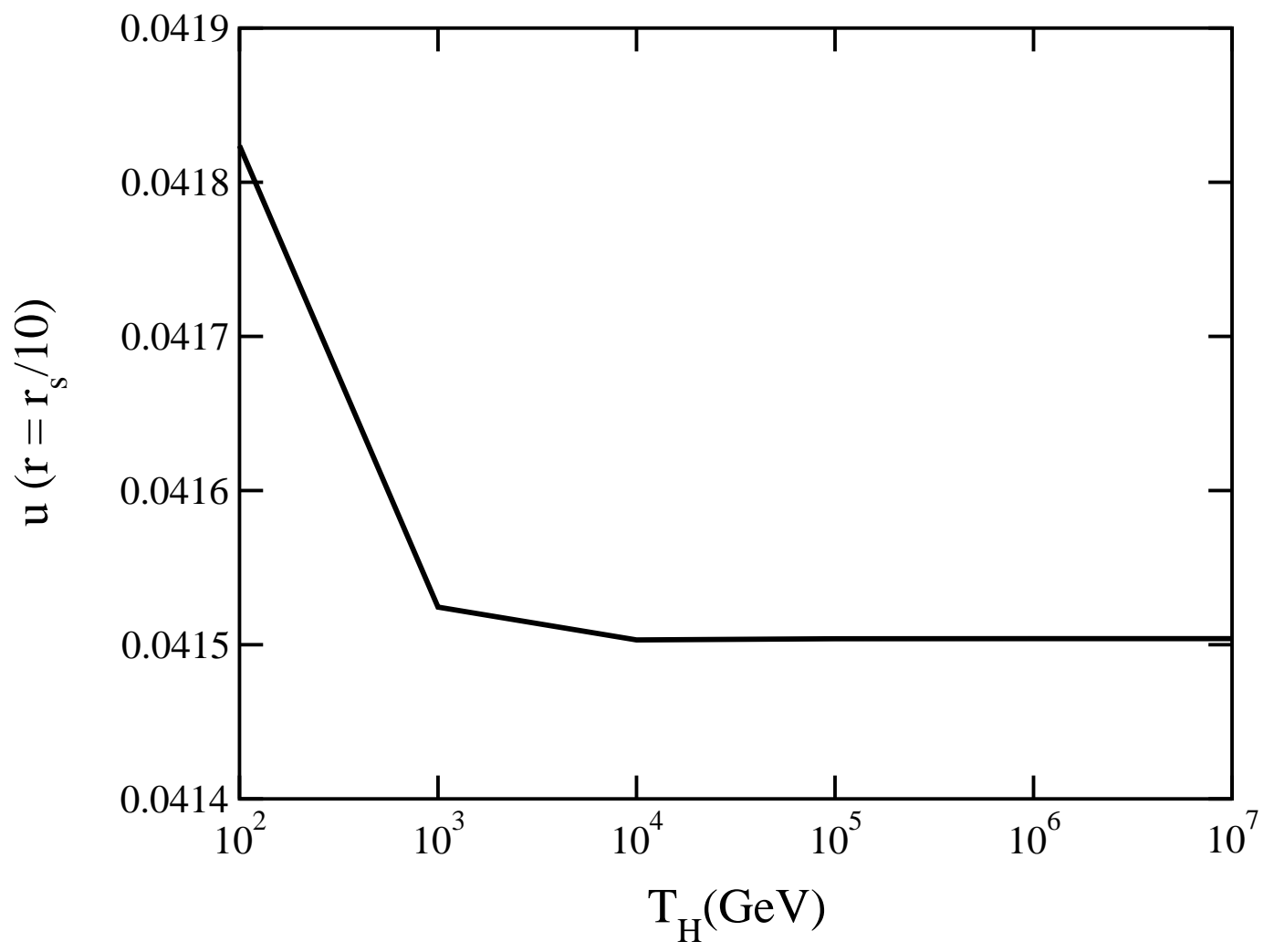

Figure 2: The value of $u=v \gamma$ at one-tenth the Schwarzschild radius as determined by numerical solution. The physical applicability of the numerical solution begins at radii greater than $r_{S}$. 


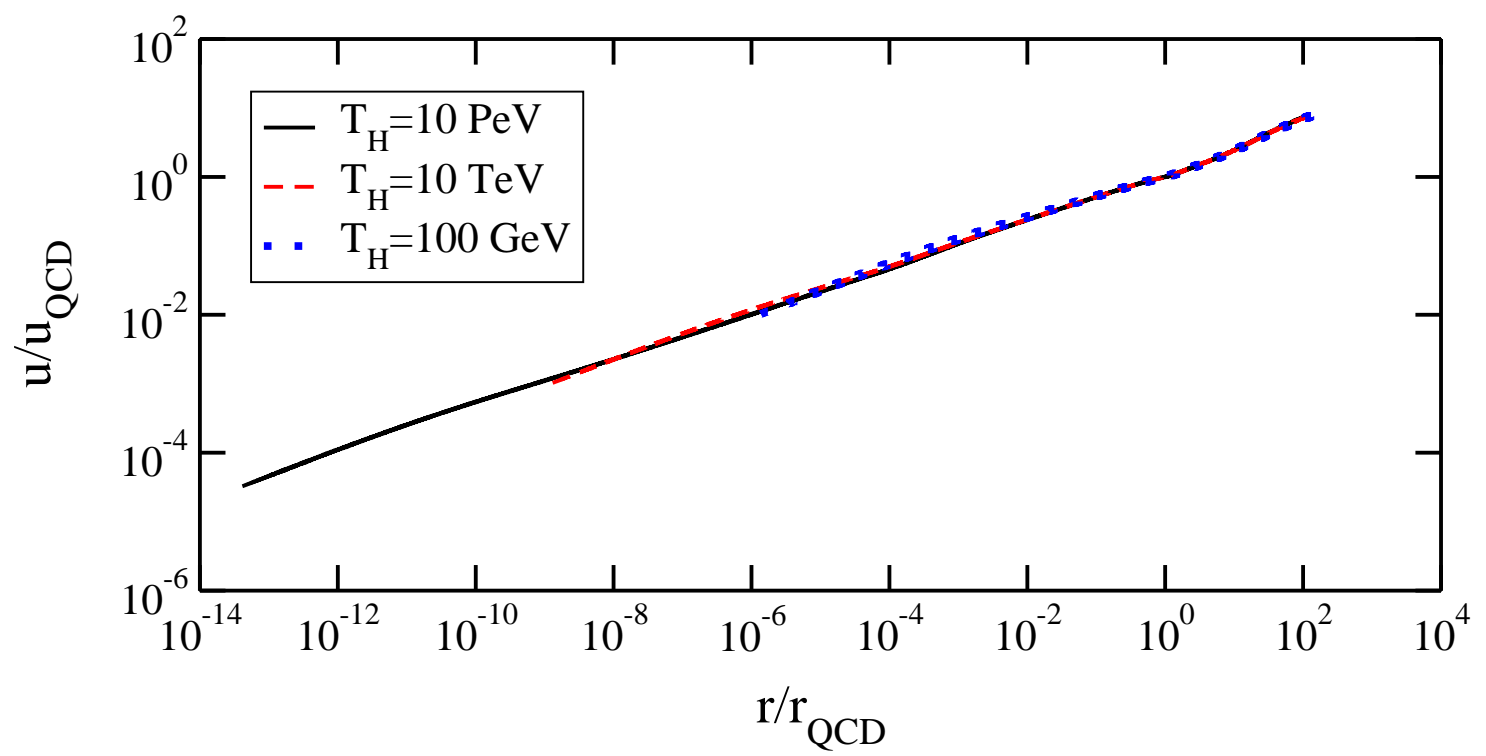

Figure 3: The radial dependence of $u$ for three different Hawking temperatures. The curves begin at $r_{S} / 10$ and terminate when the local temperature reaches $10 \mathrm{MeV}$. 


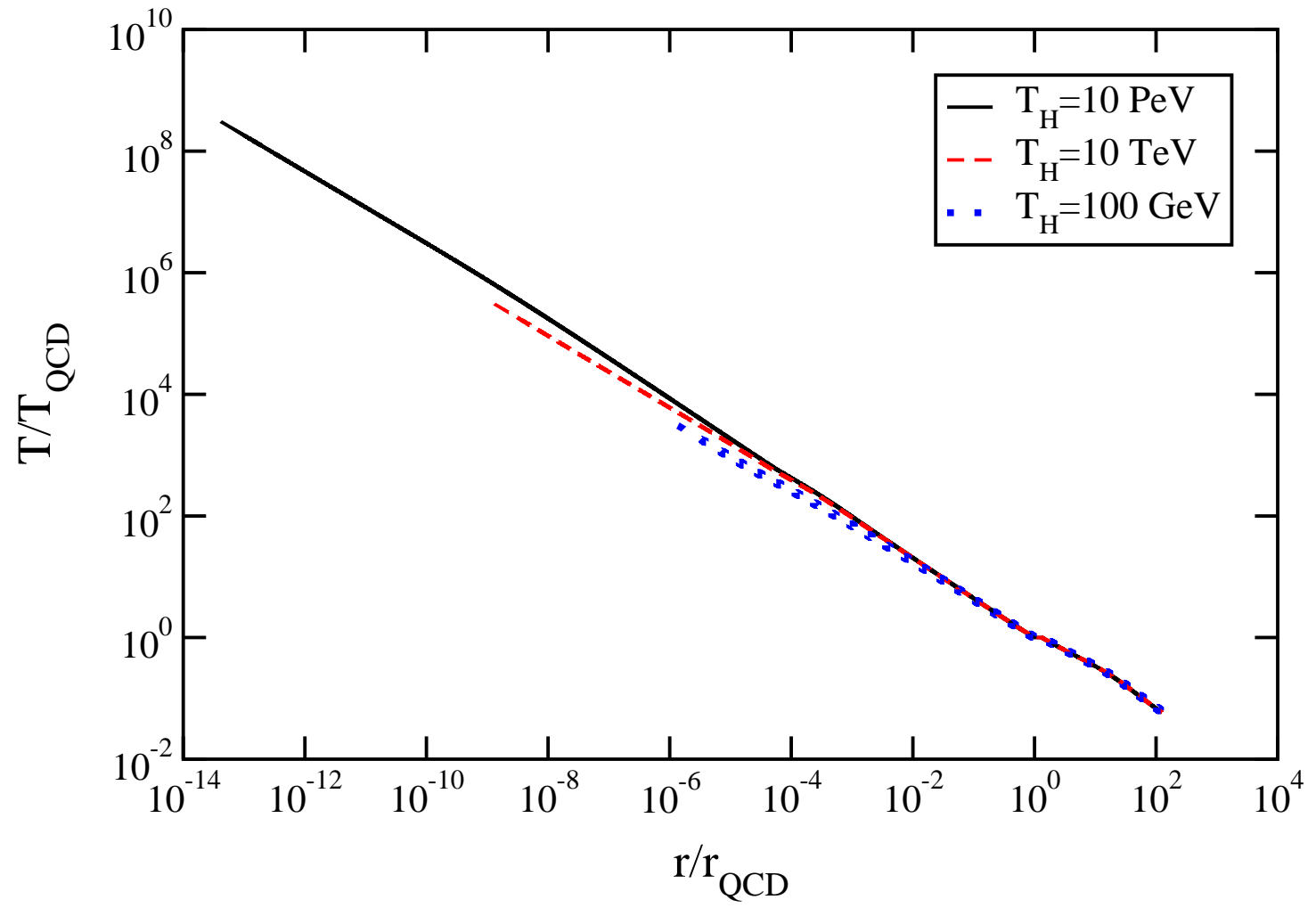

Figure 4: The radial dependence of $T$ for three different Hawking temperatures. The curves begin at $r_{S} / 10$ and terminate when the local temperature reaches $10 \mathrm{MeV}$. 


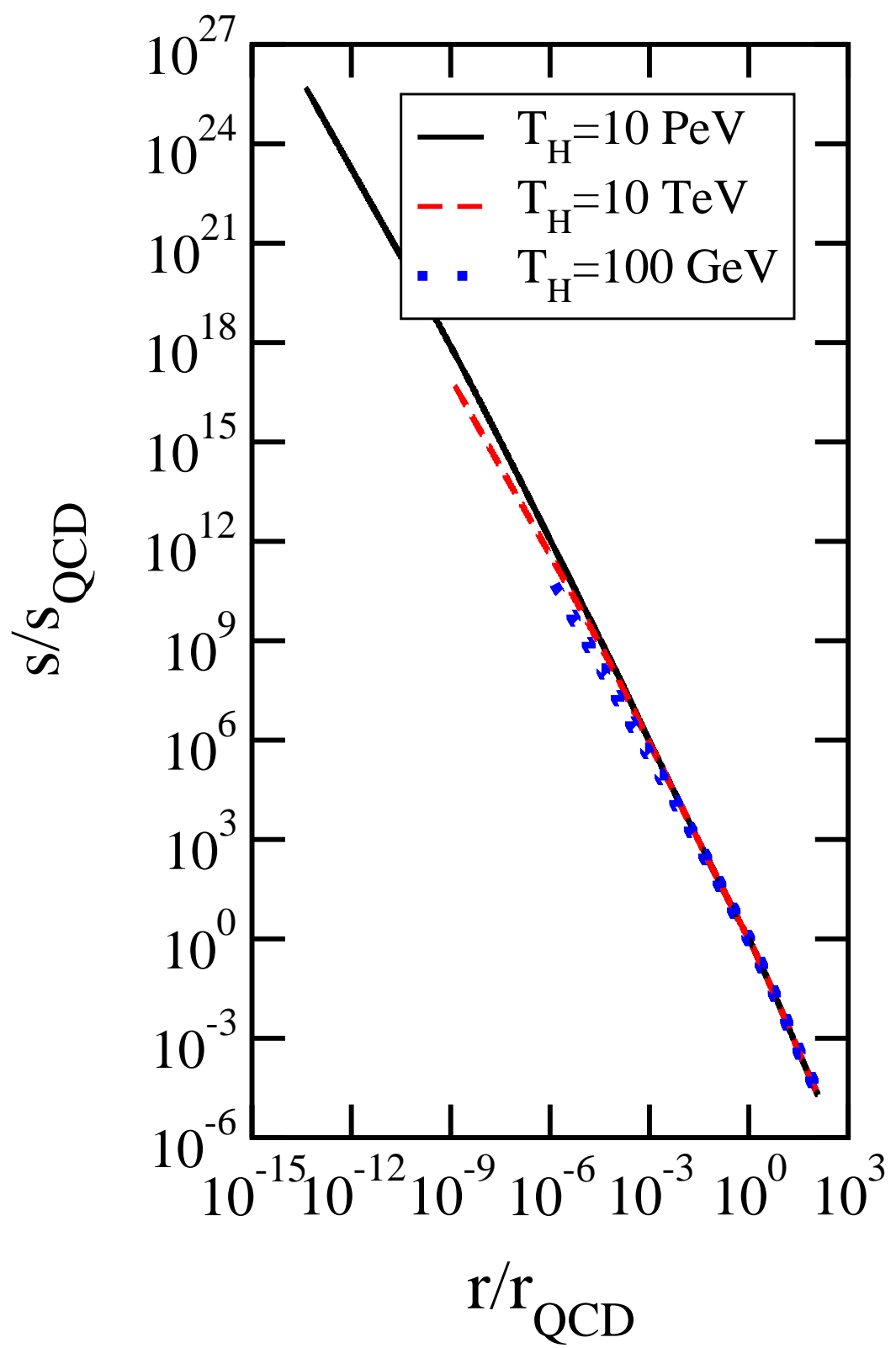

Figure 5: The radial dependence of $s$ for three different Hawking temperatures. The curves begin at $r_{S} / 10$ and terminate when the local temperature reaches $10 \mathrm{MeV}$. 


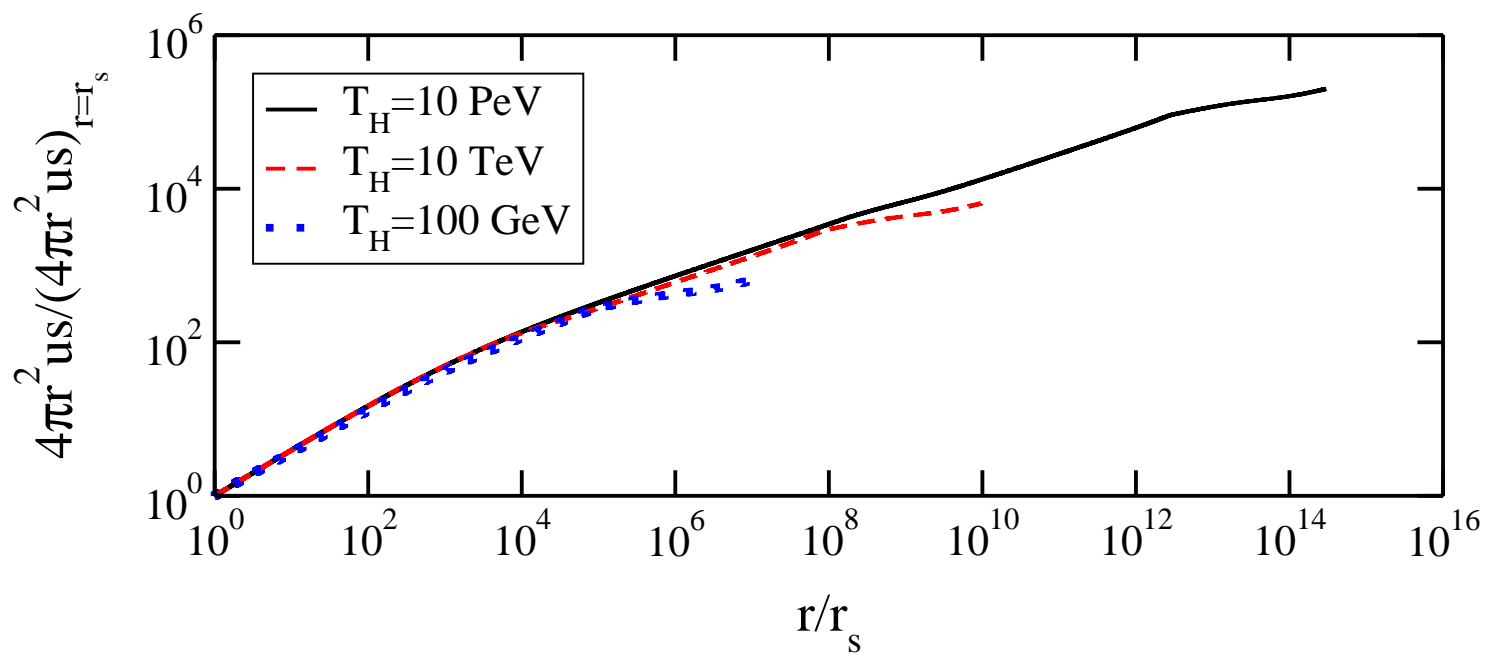

Figure 6: The radial dependence of the entropy flow for three different Hawking temperatures. The curves begin at $r_{S} / 10$ and terminate when the local temperature reaches 10 $\mathrm{MeV}$. 


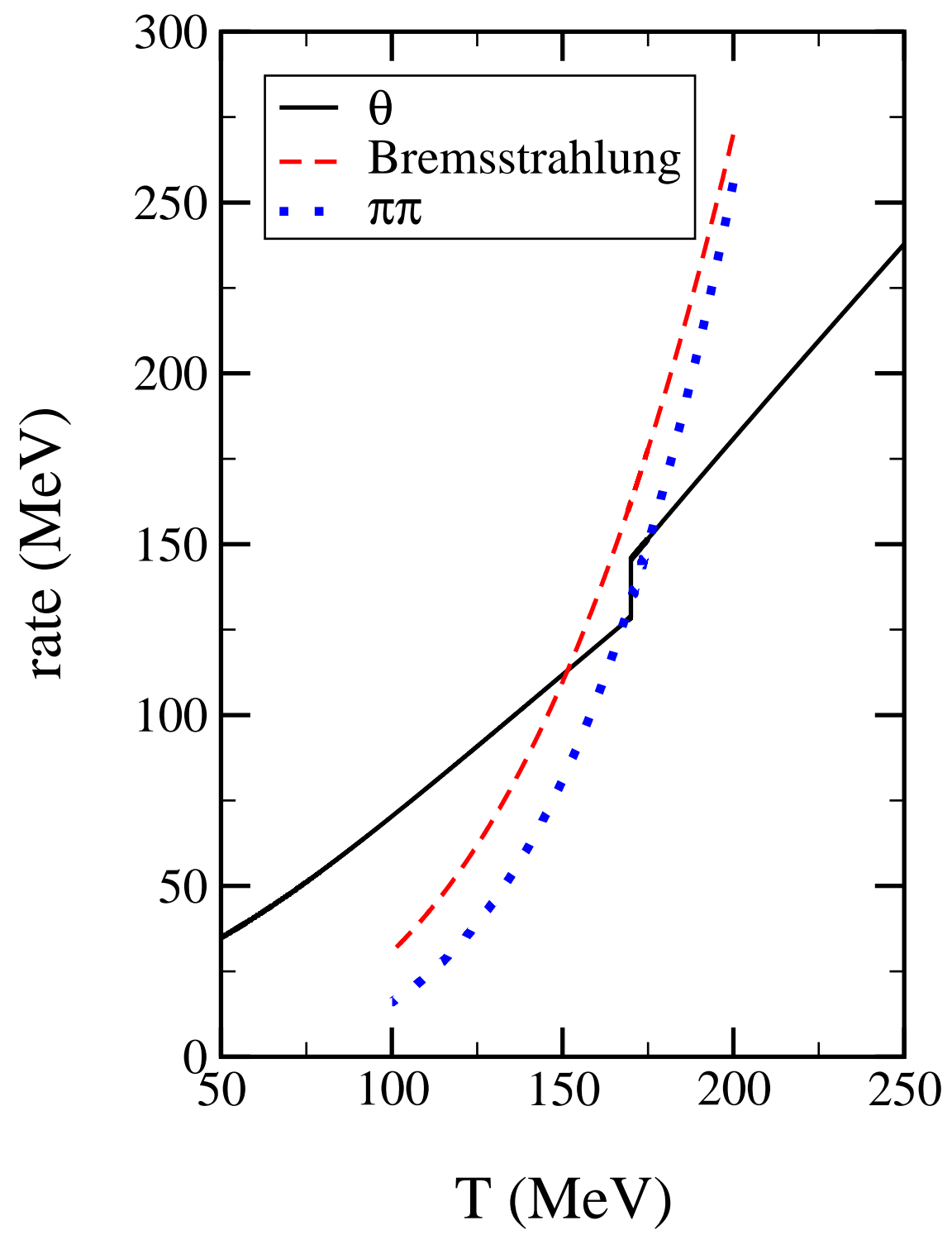

Figure 7: The rate for $\pi \pi$ scattering and for the bremsstrahlung reaction ee $\rightarrow$ ee $\gamma$ are compared to the local volume expansion rate. The Hawking temperature is $10 \mathrm{TeV}$. 


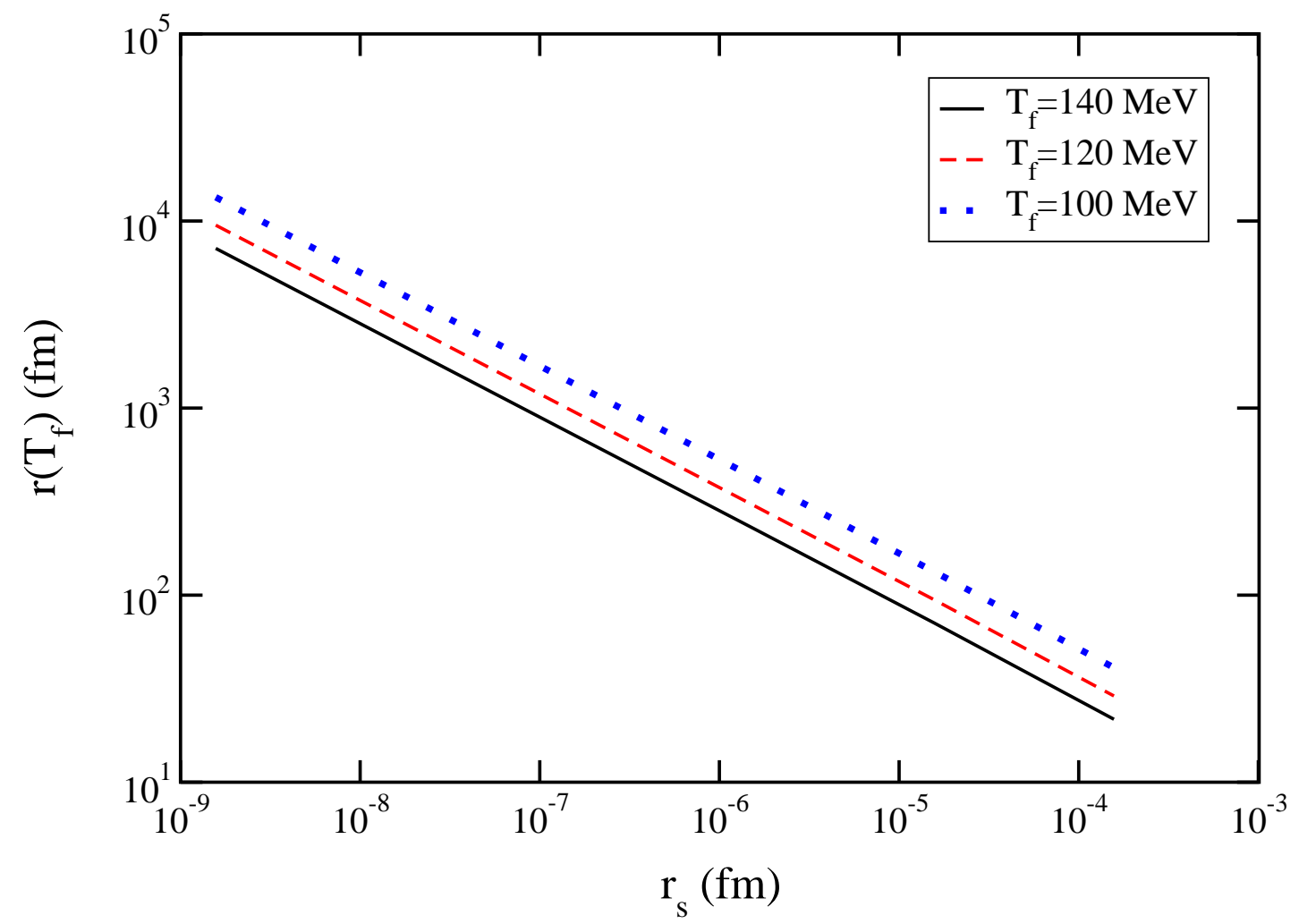

Figure 8: The freeze-out or free-streaming radius as a function of the Schwarzschild radius for three different freeze-out temperatures. 


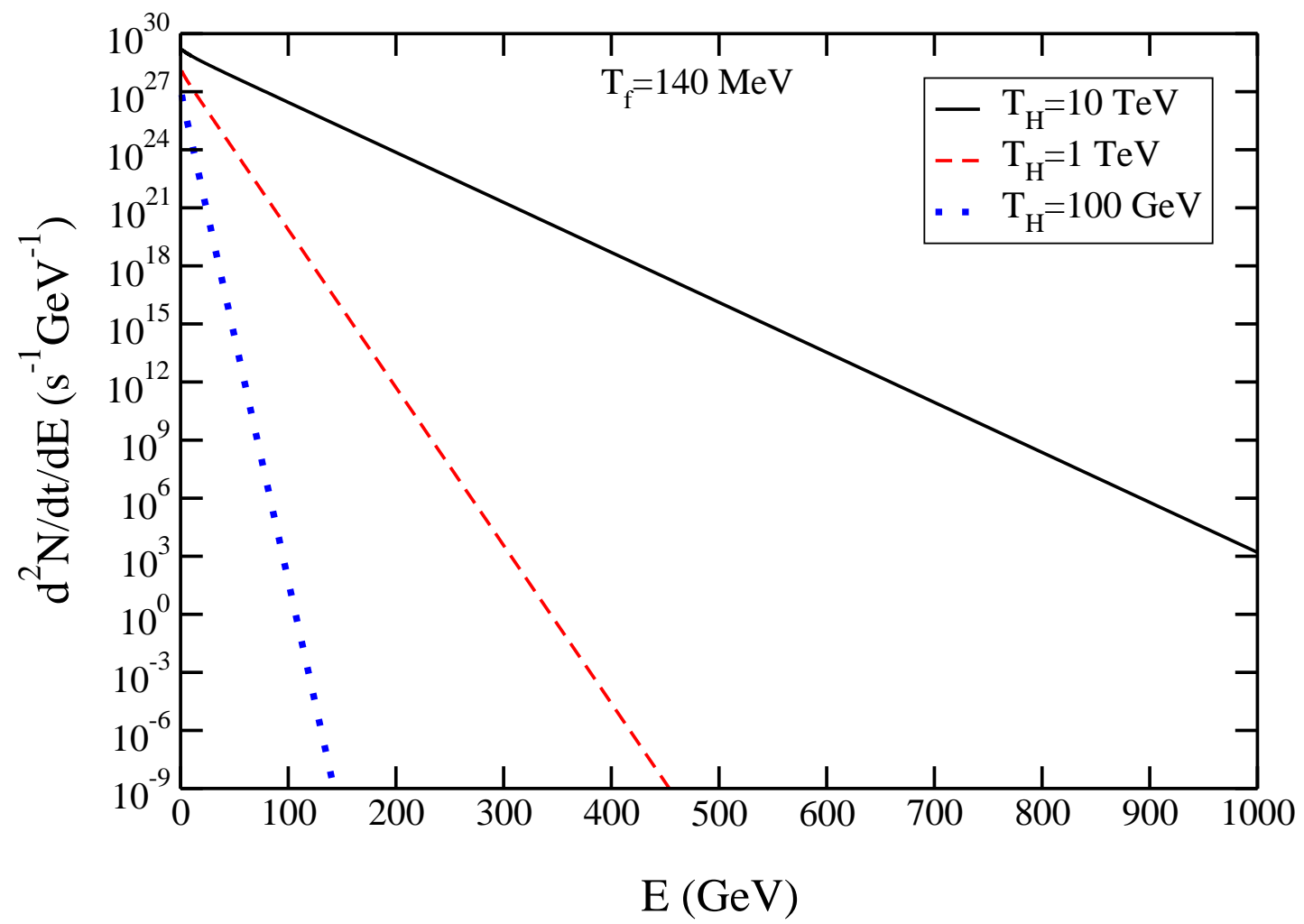

Figure 9: The instantaneous gamma ray spectrum for three different Hawking temperatures assuming $T_{f}=140 \mathrm{MeV}$. 


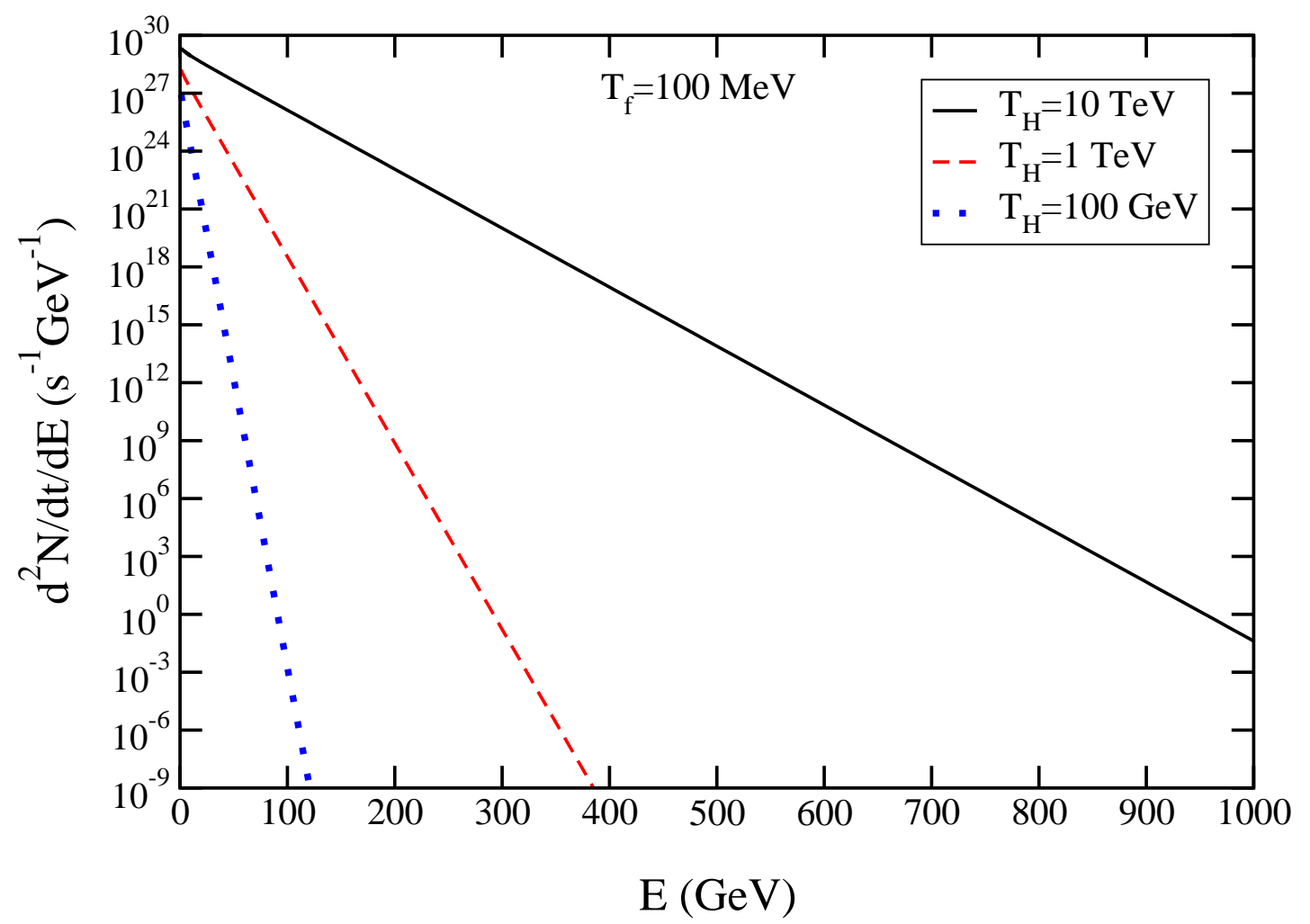

Figure 10: Same as figure 9 but with $T_{f}=100 \mathrm{MeV}$. 


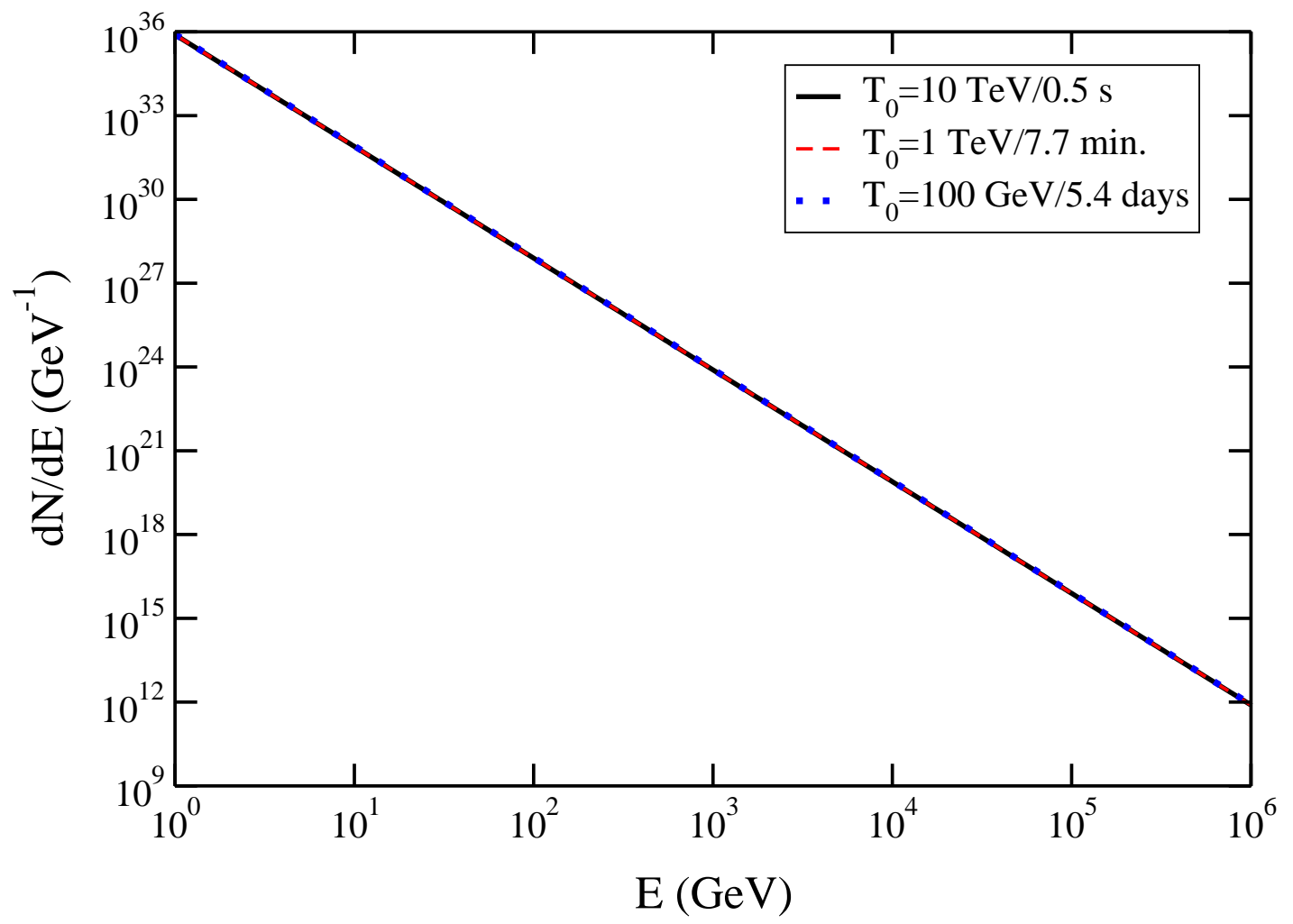

Figure 11: The time-integrated gamma ray spectrum starting from the indicated Hawking temperature. Here $T_{f}=140 \mathrm{MeV}$. 


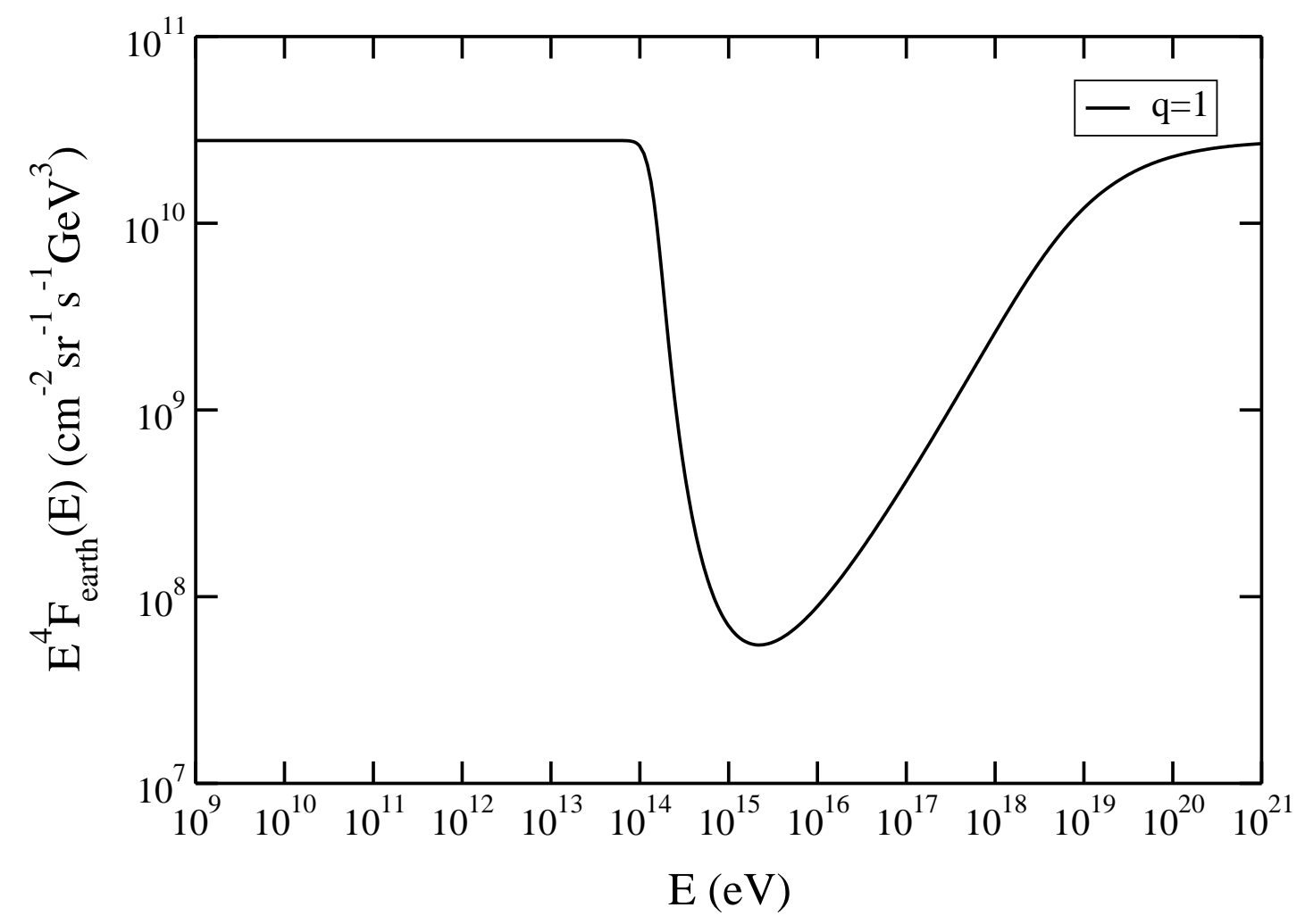

Figure 12: The flux of diffuse gamma rays coming from our galactic halo. The normalization is $\dot{\rho}_{0}=1 \mathrm{pc}^{-3} \mathrm{yr}^{-1}$. The halo is assumed to be spherically symmetric, $q=1$; the results for a flattened halo with $q=2$ are very similar. 


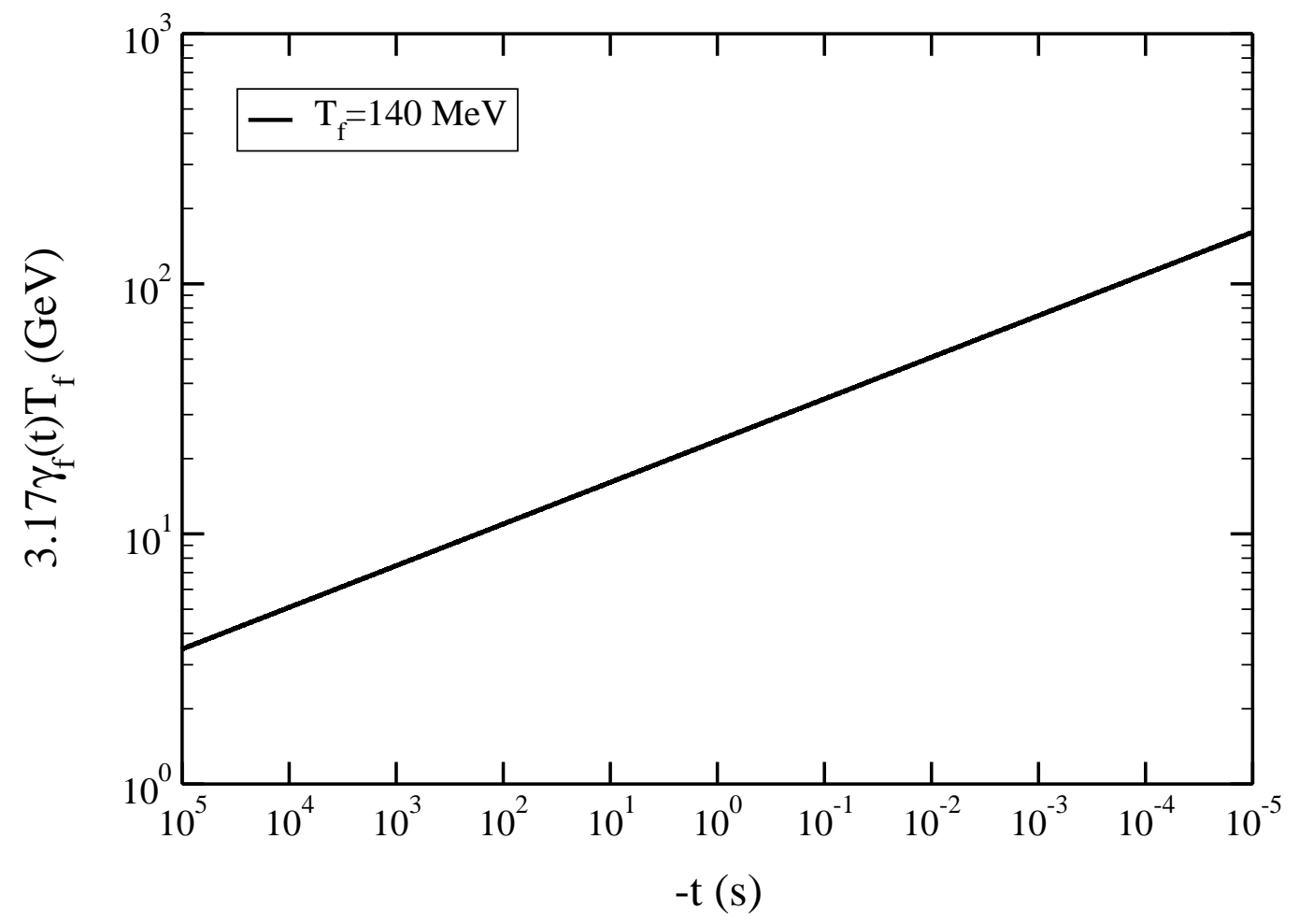

Figure 13: The average gamma ray energy as a function of the remaining lifetime of the black hole. The times spanned correspond to approximately $400 \mathrm{GeV}<T_{H}<200 \mathrm{TeV}$. 